\title{
Author Index Vol. 13,1995
}

Agrawal,R.M. 251 Alvarez, S. 190 Alvarez-Elcoro, S. 389 Banner, B.F. 223 Barton, A.L. 223

Bendtzen,K. 322 Bernsdorff, K.R. 251 Bjorkman, DJ. 119 Bloomer, J.R. 379 Bonkovsky, H.L. 223 Bourges, H. 136 Brenna, E. 25 Brodmerkel, G.J., Jr. 251 Brynskov, J. 322 Castell,D.O. 145 Cerra,F.B. 379 Chen,J.D.Z. 275 Cotton, P.B. 130 DiPalma,J $\Lambda .182$ Dyck, W.P. 92 Eusufzai, S. 312 Fantry,G.T. 108 Farrell,FJ. 291 Hellinger, W.C. 190 Hu,M.Y. 379 Jackson, B.K. 301 Jacobson, E.D. 3 Jaffe,P.E. 39 James, S.P. 108 Jeffers,L.J. 199 Keeffe,E.B. 291 Kimmey,M.B. 119 Kleveland, P.M. 25 Lee,J.G. 130 Leite,L.P. 145 Leung, J.W. 130 Levine, J.S. 3 Livingstone, A.S. 56 McCallum, R.W. 275 McCormick, S.E. 68

McGuire,B.M. 379 Maton, P. 1 Muñoz, R. 136 Nielsen, O.H. 322 Nord,HJ. 267 Nostrant, T.T. 143,337 Nyberg,S.L. 379 O’Brien,B.L. 182 Pan,J. 275 Pedersen, G. 322 Plumer,A.R. 365 Poo,J.L. 136 Ramsey, W.H. 81 Rawls,D.E. 92 Reddy,K.R. 199 Rodriguez, F. 136 RosasRomero, R. 136 Rubin, R.B. 223 Sandvik,A.K. 25 Sanyal,A.J. 153 Schiff,E.R. 199 Shaw-Stiffel, T. 239 Shiffman, M.L. 153 Sielaff,T.D. 379 Silencio, J.L. 136 Sninsky,C.A. 163 Sosa, J.L. 56 Stack, P.E. 356 Stål, P. 205 Thomas, E. 356 Tobkes,A.I. 267 Uribe,M. 136 Verne, G.N. 163 Waldum, H.L. 25 Wiesen,S. 199 Wong,R.K.H. 68 Wu,G.Y. 81 Ziebell,J. 239 Zimbalist, E.H. 365 Zurita,V.F. 92 